\title{
Robust auto-intelligent sliding accuracy for high sensitive surgical joints
}

\begin{abstract}
The objective of this paper is to design and coordinate controllers that will enhance transient stability of three dimensions motor subject to large disturbances. Two specific classes of controllers have been investigated, the first one is a type of disturbance signals added to the excitation systems of the generating units. To address a wide range of operating conditions, a nonlinear control design technique, called highly nonlinear computed torque control, is used. While these two types of controllers improve the dynamic performance significantly, a coordination of these controllers is even more promising. Results show that the proposed control technique provides better stability than conventional computed torque fixed gain controllers.
\end{abstract}

Keyword: Intelligent methodology; Medical application; Online tuning; PI fuzzy logic theory; Sliding surface slope; Surgical robots; Variable structure controller 